\title{
Pharmacological differentiation of opioid receptor antagonists by molecular and functional imaging of target occupancy and food reward-related brain activation in humans
}

\author{
EA Rabiner 1,2,8 J Beaver $^{1,8}$, A Makwana1, G Searle1, C Long ${ }^{1}$, PJ Nathan ${ }^{3,4}$, RD Newbould ${ }^{1}$, J Howard ${ }^{1}$, \\ SR Miller ${ }^{5}$, MA Bush ${ }^{6}$, S Hill' ${ }^{1}$, R Reiley ${ }^{1}$, J Passchier ${ }^{1}$, RN Gunn ${ }^{1,2,7}$, PM Matthews ${ }^{1,2}$ and ET Bullmore B $^{3,4}$ \\ ${ }^{1}$ GSK Clinical Imaging Centre, Imperial College London, Hammersmith Hospital, London, UK; ${ }^{2}$ Centre for Neurosciences, \\ Division of Experimental Medicine and Toxicology, Imperial College, London, UK; ${ }^{3}$ GSK Academic Discovery Performance Unit \\ and Clinical Unit Cambridge, Addenbrooke's Centre for Clinical Investigations, Cambridge Biomedical Campus, Cambridge, \\ UK; ${ }^{4}$ Behavioural and Clinical Neuroscience Institute, Department of Psychiatry, University of Cambridge, Cambridge, UK; \\ ${ }^{5}$ GSK Quantitative Sciences, Stevenage, UK; ${ }^{6}$ GSK Clinical Pharmacology Modeling and Simulation, Research Triangle Park, \\ NC, USA and ${ }^{7}$ Department of Engineering Science, University of Oxford, Oxford, UK
}

\begin{abstract}
Opioid neurotransmission has a key role in mediating reward-related behaviours. Opioid receptor (OR) antagonists, such as naltrexone (NTX), can attenuate the behaviour-reinforcing effects of primary (food) and secondary rewards. GSK1521498 is a novel OR ligand, which behaves as an inverse agonist at the $\mu$-OR sub-type. In a sample of healthy volunteers, we used $\left[{ }^{11} \mathrm{C}\right]$-carfentanil positron emission tomography to measure the OR occupancy and functional magnetic resonance imaging (fMRI) to measure activation of brain reward centres by palatable food stimuli before and after single oral doses of GSK1521498 (range, 0.4-100 mg) or NTX (range, 2-50 mg). GSK1521498 had high affinity for human brain ORs (GSK1521498 effective concentration $50=7.10 \mathrm{ng} \mathrm{m}^{-1}$ ) and there was a direct relationship between receptor occupancy (RO) and plasma concentrations of GSK1521498. However, for both NTX and its principal active metabolite in humans, 6- $\beta-N T X$, this relationship was indirect. GSK1521498, but not NTX, significantly attenuated the fMRI activation of the amygdala by a palatable food stimulus. We thus have shown how the pharmacological properties of OR antagonists can be characterised directly in humans by a novel integration of molecular and functional neuroimaging techniques. GSK1521498 was differentiated from NTX in terms of its pharmacokinetics, target affinity, plasma concentration-RO relationships and pharmacodynamic effects on food reward processing in the brain. Pharmacological differentiation of these molecules suggests that they may have different therapeutic profiles for treatment of overeating and other disorders of compulsive consumption.

Molecular Psychiatry (2011) 16, 826-835; doi:10.1038/mp.2011.29; published online 19 April 2011
\end{abstract}

Keywords: positron emission tomography; functional MRI; experimental medicine; pharmacokinetics; pharmacodynamics; neuropharmacology

\section{Introduction}

The endogenous opioid neurotransmitter system comprises a number of peptides (including $\beta$-endorphins, enkephalins and dynorphins) and three major opioid receptor (OR) sub-types: $\mu, \delta$ and $\kappa^{1-3}$ OR agonists increase, whereas antagonists decrease, feeding and other rewarding behaviours in animal

Correspondence: Professor E Bullmore, GSK Academic Discovery Performance Unit and Clinical Unit Cambridge, Addenbrooke's Centre for Clinical Investigations, Cambridge Biomedical Campus, Cambridge CB2 0GG, UK.

E-mail: edward.t.bullmore@gsk.com

${ }^{8}$ These authors contributed equally to this work.

Received 12 January 2011; revised 16 February 2011; accepted 24 February 2011; published online 19 April 2011 models. ${ }^{4-9}$ Agonism at the $\mu$-OR sub-type seems to be particularly effective in enhancing the hedonic and consummatory eating behaviours, ${ }^{6,10-12}$ as demonstrated by greater intake of energy-dense foods. This effect is blocked by OR antagonists. ${ }^{13,14}$ Direct infusion of $\mu$-OR agonists has been used to localise effects on eating behaviour to the nucleus accumbens in rats. ${ }^{10-13,15,16}$ The basal nucleus of the amygdala provides a major input to the nucleus accumbens, ${ }^{17}$ amplifying the hedonic value of palatable food and its motivational influence on goal-directed

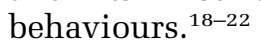

Opioid signalling in humans has been implicated in behaviours reinforced by primary rewards, especially fatty or sugary foods, or secondary rewards, such as self-administered drugs. Acute administration of 
drugs broadly classified as OR antagonists (for example, naltrexone (NTX)) moderately reduces short-term food intake ${ }^{23}$ and affective or subjective pleasantness of palatable foods in healthy subjects; ${ }^{23-26}$ and reduces food or drug intake in short-term animal and experimental medicine models of obesity, binge eating, alcohol and drug dependence syndromes. ${ }^{23-28}$ NTX is licensed for treatment of alcohol and opiate addiction.

GSK1521498 is a novel OR ligand with a high degree of selectivity for the $\mu$-OR sub-type in binding studies (Table 1). It has antagonist effects at the $\mu-\mathrm{OR}$ receptor, or inverse agonist properties under conditions of constitutive receptor activity. In a first-inhuman acute dose escalation study, single doses were safe and well tolerated up to a maximum tolerated dose of $100 \mathrm{mg} .{ }^{33}$ A single $25 \mathrm{mg}$ dose of GSK1521498 was associated with reduced pleasurable response to, and reduced consumption of, high fat/high sugar snack items in an experimental model of overeating behaviour in overweight volunteers. ${ }^{33}$

Here we used neuroimaging to investigate $\mu-\mathrm{OR}$ occupancy and effects on brain function of single doses of NTX and GSK1521498 in healthy volunteers. We used positron emission tomography (PET) with $\left[{ }^{11} \mathrm{C}\right]$-carfentanil to measure $\mu$-OR occupancy over a range of doses and plasma concentrations of both drugs. In the same scanning sessions, we also used functional magnetic resonance imaging (fMRI) to measure food reward-related activation of theoretically predicted brain regions, including amygdala and striatum. ${ }^{34-39}$ We compared the two drugs in terms of their pharmacokinetics, target occupancy and proximal functional efficacy.

\section{Materials and methods}

\section{Participants}

In all, 26 healthy male volunteers (25-60 years old, body weight $>50 \mathrm{~kg}$ and body mass index 19$30 \mathrm{~kg} \mathrm{~m}^{-2}$ ) were recruited from the London area by a research organisation, Hammersmith Medical Research, contracted by the GSK Clinical Imaging Centre, London. All subjects satisfied eligibility criteria and passed a medical screen for fitness to participate (see Supplementary Information for details), and provided informed consent for participation in writing.

\section{Study design}

This was an open label study, with participants assigned to either GSK1521498 or NTX treatment

Table 1 Opioid receptor sub-type binding affinities and plasma pharmacokinetic parameters for naltrexone, 6- $\beta$-naltrexol and GSK1521498

\begin{tabular}{|c|c|c|c|}
\hline \multicolumn{4}{|c|}{ OR sub-type affinity (nM) } \\
\hline & Naltrexone & 6 - $\beta$-Naltrexol & GSK1521498 \\
\hline \multirow[t]{4}{*}{$\mu-\mathrm{OR}$} & 0.5 (ref. 29) & 1.4 (ref. 29) & \\
\hline & & 2.1 (ref. 30) & \\
\hline & 0.31 (ref. 31) & 0.74 (ref. 31) & \\
\hline & $4.7^{\mathrm{a}}$ & & $1.5^{\mathrm{a}}$ \\
\hline \multirow[t]{3}{*}{$\kappa-\mathrm{OR}$} & 1.0 (ref. 29) & 2.0 (ref. 29) & \\
\hline & & 7.4 (ref. 30) & \\
\hline & $20.0^{\mathrm{a}}$ & & $20.4^{\mathrm{a}}$ \\
\hline \multirow[t]{2}{*}{$\delta$-OR } & 7.0 (ref. 29) & $\begin{array}{l}29 \text { (ref. 29) } \\
213 \text { (ref. 30) }\end{array}$ & \\
\hline & $42^{\mathrm{a}}$ & & $30.2^{\mathrm{a}}$ \\
\hline \multicolumn{4}{|c|}{ Pharmacokinetic parameters (minimum-maximum) } \\
\hline $\operatorname{AUC}(0, t), \mathrm{ngh}^{-1} \mathrm{ml}^{-1}$ & $0.06-23.6$ & $14.1-618$ & $20.4-10422$ \\
\hline AUC $(0, t), \mathrm{nMh}^{-1}$ & $0.18-69.1$ & $41-1800$ & $50.7-25842$ \\
\hline$C_{\max }, \mathrm{ng} \mathrm{ml}^{-1}$ & $0.046-9.01$ & $1.2-93$ & $1.5-929$ \\
\hline$C_{\max }, \mathrm{nM}$ & $0.13-26.4$ & $3.5-271$ & $3.8-2308$ \\
\hline Exposures during PET scanning, $\mathrm{ng} \mathrm{ml}^{-1}$ & $0.013-1.87$ & $0.32-37.8$ & $1.1-286$ \\
\hline Exposures during PET scanning, nM & $0.04-5.5$ & $0.94-110$ & $2.7-711$ \\
\hline$T_{\max }, \mathrm{h}$ & $0.5-2$ & $0.5-2$ & $1-4$ \\
\hline $\mathrm{T} 1 / 2, \mathrm{~h}$ & 4 (ref. 32) & 13 (ref. 32) & 20-24 (ref. 33) \\
\hline
\end{tabular}

Abbreviations: AUC, area under curve; OR, opioid receptor; PET, positron emission tomography.

${ }^{\mathrm{a}}$ GlaxoSmithKline internal data.

While data from different studies can be used to compare drug selectivities at the OR sub-types, comparison of absolute affinity values from different studies should be carried out cautiously because of the differences in the system examined and assay conditions. Affinity data were estimated from $\mathrm{pKi}$ of displacement of appropriate radio ligands from human ORs expressed in a cell system, ${ }^{29,30}$ or from monkey cortical homogenates. ${ }^{31}$ The GlaxoSmithKline internal data were estimated from a functional (f)pKi in a $\left[{ }^{35} \mathrm{~S}\right] \mathrm{GTP} \gamma \mathrm{S}$ assay on human OR expressed in CHO-E1A cells.

$\mathrm{pKi}=$ base 10 logarithm of the drug concentration that gives $50 \%$ of maximum receptor binding. 
groups at enrolment. Each participant underwent up to three $\left[{ }^{11} \mathrm{C}\right]$-carfentanil PET scans and two fMRI examinations: one $\left[{ }^{11} \mathrm{C}\right]$-carfentanil PET scan and one fMRI scan at baseline (before dosing) and up to two PET scans and one fMRI scan following oral administration of a single dose of GSK1521498 or NTX. Two participants opted to withdraw from the study following completion of the baseline scanning session: the complete PET dataset available for analysis therefore comprised 24 participants.

The administered doses of GSK1521498 or NTX were chosen adaptively to optimise the estimation of the dose-occupancy relationship for each drug on the basis of data acquired from the preceding examinations in the study. ${ }^{40}$ The administered dose range was 0.4-100 mg for GSK1521498, and 2-50 mg for NTX. The maximum doses administered were equal to the maximum tolerated dose of GSK1521498 determined in the first-in-human study ${ }^{33}$ and the standard clinical dose of NTX used for alcohol dependence. ${ }^{41}$

The times and doses of the two post-dose $\left[{ }^{11} \mathrm{C}\right]-$ carfentanil PET scans were chosen adaptively for each subject to optimise estimation of the relationship between plasma concentration and RO. ${ }^{40}$ Post-dose $\left[{ }^{11} \mathrm{C}\right]$-carfentanil PET scans were acquired at $3-36 \mathrm{~h}$ after the administration of GSK1521498 and at 3-88 h after the administration of NTX. Post-dose fMRI scans were acquired within $60 \mathrm{~min}$ of the first post-dose PET scan.

Sampling and analysis of drug plasma concentrations Venous blood samples were collected at regular intervals throughout the scanning sessions. Highperformance liquid chromatography/mass spectrometry/mass spectrometry was used to estimate the plasma concentrations of GSK1521498, NTX, and the major metabolite of NTX, 6- $\beta$-naltrexol (6- $\beta$-NTX; see Supplementary Information for assay details). Drug plasma concentration at the start of each PET scan was used to model the relationship between drug concentrations and $\mu-\mathrm{OR}$ occupancies.

\section{[11C]-carfentanil PET data acquisition and pre-processing}

Carfentanil (methyl 1-(2-phenylethyl)-4-(phenyl (propanoyl)amino)-4-piperidinecarboxylate 3S, 5S; Advanced Biochemical Compounds, Radeberg, Germany), a potent selective $\mu$-OR agonist, was labelled with carbon-11 using a modification of a previously described method ${ }^{42}$ implemented using a semiautomated Modular Lab Multifunctional Synthetic Module (Eckert \& Ziegler, Berlin, Germany). The final product was reformulated in sterile $0.9 \%$ saline containing $\sim 10 \%$ ethanol (v/v) and satisfied quality control criteria for specific activity and purity before being injected intravenously as a slow bolus over $\sim 30 \mathrm{~s}$.

PET scanning was conducted in three-dimensional mode using a Siemens Biograph 6 Hi-Rez PET-CT for the NTX group and a Siemens Biograph 6 TruePoint PET-CT for the GSK1521498 group (Siemens Healthcare, Erlangen, Germany). A low-dose CT scan was acquired for attenuation correction before the administration of the radiotracer. Dynamic PET data were acquired for $90 \mathrm{~min}$ after $\left[{ }^{11} \mathrm{C}\right]$-carfentanil injection, binned into 26 frames (durations: $8 \times 15 \mathrm{~s}$, $3 \times 60 \mathrm{~s}, 5 \times 2 \mathrm{~min}, 5 \times 5 \mathrm{~min}$ and $5 \times 10 \mathrm{~min})$, reconstructed using fourier re-binning and a two-dimensional-filtered back projection algorithm and then smoothed with a two-dimensional Gaussian filter ( $5 \mathrm{~mm}$ at full width half maximum).

\section{PET data analysis}

Dynamic PET images were registered to each participant's T1-weighted anatomical MRI volume and corrected for head motion using SPM5 software (Wellcome Trust Centre for Neuroimaging, http:// www.fil.ion.ucl.ac.uk/spm). Pre-selected regions of interests (ROIs; amygdala, caudate, putamen, thalamus, cerebellum, frontal and occipital cortices) were defined bilaterally on the T1-weighted anatomical volume using an in-house atlas ${ }^{43}$ and applied to the dynamic PET data to generate regional time-activity curves (Figure 1).

The $\left[{ }^{11} \mathrm{C}\right]$-carfentanil-specific binding was quantified as binding potential relative to the non-displaceable compartment $\left(\mathrm{BP}_{\mathrm{ND}}{ }^{44}\right)$

$$
\mathrm{BP}_{\mathrm{ND}}=\frac{f_{\mathrm{ND}} B_{\text {avail }}}{K_{\mathrm{D}}}
$$

where $f_{\mathrm{ND}}$ is the free fraction of the radioligand in the brain, $K_{\mathrm{D}}$ is the affinity of $\left[{ }^{11} \mathrm{C}\right]$-carfentanil, and $B_{\text {avail }}$ is the density of the available $\mu$-ORs. Regional $\left[{ }^{11} \mathrm{C}\right]-$ carfentanil $\mathrm{BP}_{\mathrm{ND}}$ was estimated using a reference tissue $\operatorname{model}^{45}$ with the occipital cortex as the reference region. Drug related occupancy of the $\mu$-OR was quantified as a reduction of $\left[{ }^{11} \mathrm{C}\right]$-carfentanil $\mathrm{BP}_{\mathrm{ND}}$

$$
\text { Occupancy }_{\text {Drug }}=\frac{\mathrm{BP}_{\mathrm{ND}}^{\text {Baseline }}-\mathrm{BP}_{\mathrm{ND}}^{\text {Drug }}}{\mathrm{BP}_{\mathrm{ND}}^{\text {Baseline }}}
$$

The affinity constant for each drug at the $\mu$-OR (effective concentration $50\left(\mathrm{EC}_{50}\right)$ ) was estimated by fitting the plasma concentration measured at the start of the PET scan, $C_{\text {Drug }}^{P}$ to the estimated occupancy:

$$
\text { Occupancy }_{\text {Drug }}=\frac{C_{\text {Drug }}^{P}}{C_{\text {Drug }}^{P}+\mathrm{EC}_{50}}
$$

\section{fMRI: food stimuli and activation paradigm}

Food stimuli for the fMRI task were delivered orally in the scanner and, for different trials, were either $0.5 \mathrm{ml}$ of a highly palatable commercially available drink (marketed in the United Kingdom as a 'smoothie'), $0.5 \mathrm{ml}$ of a neutral solution (25 mM KCL and $2.5 \mathrm{mM} \mathrm{NaHCO}_{3}$ in water) or $1.0 \mathrm{ml}$ of purified water. Although data from trials with the neutral solution will not be reported here, the full activation paradigm consisted of 16 palatable drink and 16 neutral solution trials, presented in randomised order. Purified water was delivered after each 
a
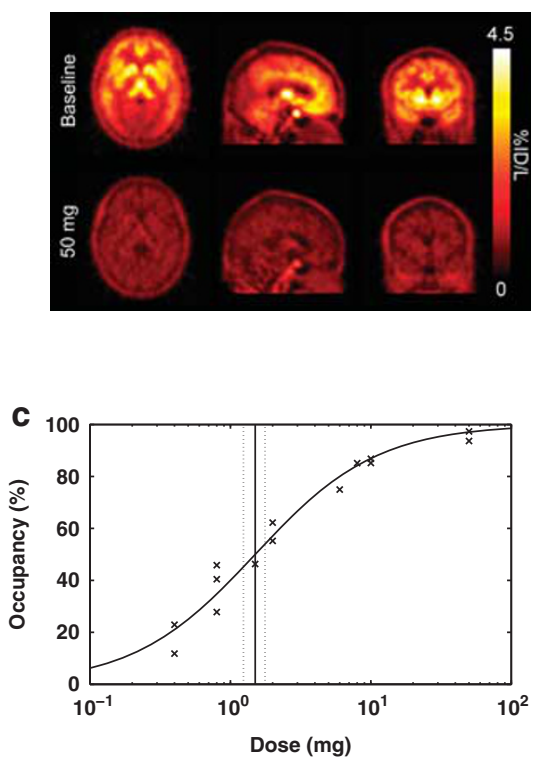

b
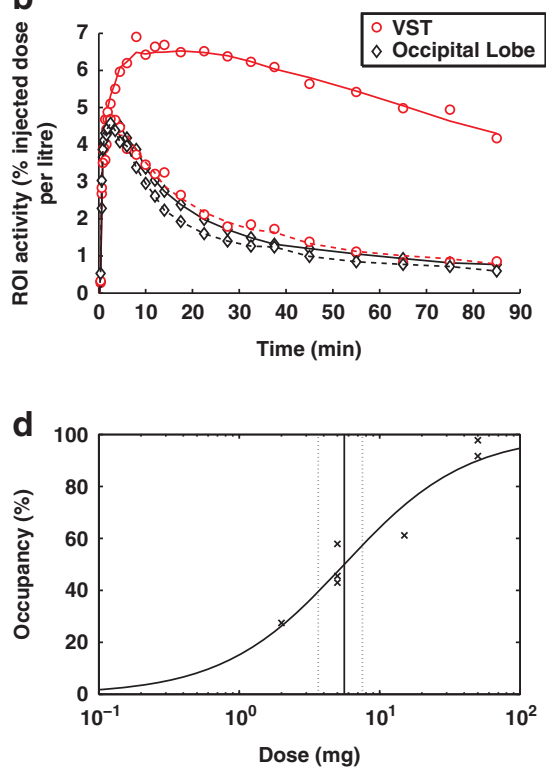

Figure 1 Opioid receptor binding by $\left[{ }^{11} \mathrm{C}\right]$-carfentanil and its displacement by naltrexone and GSK1521498. (a) Slices of an individual positron emission tomography scan demonstrating high binding in sub-cortical nuclei at baseline, which was reduced following administration of GSK1521498 $50 \mathrm{mg}$. (b) Time-activity curves for ventral striatum (VST, red lines) and for occipital cortex (black lines) at baseline (solid lines) and after a dose of GSK1521498 50 mg (broken lines). Dose-occupancy curves for (c) GSK1521498 and (d) naltrexone, estimated from scans acquired at $<8 \mathrm{~h}$ after dosing. Vertical line indicates effective dose 50 and the dotted lines are its $95 \%$ confidence interval.

stimulus trial; we allowed a slightly greater volume of purified water $(1 \mathrm{ml})$ to wash away residual traces of the flavoured stimuli $(0.5 \mathrm{ml})$ between trials. To minimise head movements during delivery of the stimuli, participants were instructed to refrain from swallowing until a cue was presented $5 \mathrm{~s}$ later.

\section{fMRI: data acquisition}

T2*-weighted, dual echo, echo-planar images sensitive to blood oxygenation level-dependent (BOLD) contrast were acquired continuously on a 3T Siemens Tim Trio scanner with a 32-channel head coil (Siemens Healthcare). Each scan session consisted of 390 volumes of a 38-slice acquisition, angled $\sim 30^{\circ}$ coronally to the anterior-posterior commissural plane to minimise signal dropout in orbitofrontal and medial temporal regions (relaxation time $=2100 \mathrm{~ms}$; dual echo time (TEs) $=13$ and $31 \mathrm{~ms}$; flip angle $=80^{\circ}$, matrix size $=64 \times 64$, and field of view $=225 \times$ $225 \mathrm{~mm}$ for voxel size of $3.0 \times 3.0 \times 3.0 \mathrm{~mm}$ ). Highresolution T1-weighted anatomical scans (ADNI MPRAGE (Alzheimer's disease neuroimaging initiative magnetization-prepared rapid gradient echo) ${ }^{46}$ ) were acquired with whole-brain coverage (208 slices) for each participant to facilitate fMRI and PET image co-registration and PET ROI definition (relaxation time $=3000 \mathrm{~ms}, \mathrm{TE}=3.66 \mathrm{~ms}$, flip angle $=9^{\circ}$, voxel size $=1 \mathrm{~mm}^{3}$ ).

\section{fMRI: data analysis}

fMRI data were corrected for head motion, high-pass filtered and spatially smoothed using FSL software: http://www.fmrib.ox.ac.uk/fsl (see Supplementary Information for details). We estimated the difference in regional mean BOLD signal intensity between periods of palatable stimulus delivery and periods of purified water delivery, at each of nine ROIs bilaterally (as defined a priori in the protocol at http://clinicaltrials.gov). These estimates were then averaged over right and left homologous regions of amygdala, caudate, globus pallidus, hippocampus, insula, nucleus accumbens, orbitofrontal cortex, thalamus and putamen. In each treatment group, we tested the null hypothesis that the within-subject difference between baseline (pre-dose) and post-dose scans in regional BOLD response to palatable stimuli versus purified water was zero: (post-dose BOLD response to palatable trials)-(pre-dose BOLD response to palatable trials $)=\Delta-B O L D=0$. To control for between-subject variability in $\mu$-OR occupancy (by either drug) and for variability in BOLD activation at baseline, we also tested $\Delta$-BOLD in each ROI using a linear model including baseline BOLD activation and $\mathrm{RO}$ as covariates. To control for multiple comparisons in these planned regional analyses of treatment-related differences in brain activation, the threshold for significance was Bonferroni corrected at $P<0.05 / 9=0.0055$. We also tested the null hypothesis that there is no difference in $\Delta$-BOLD between drugs $\left(\Delta\right.$-BOLD $_{\mathrm{GSK} 1521498}$ $\left.=\Delta-\mathrm{BOLD}_{\mathrm{NTX}}\right)$, and between-drug comparisons were controlled for baseline activation and RO.

We also conducted exploratory voxel-level analyses of the fMRI data. Combining data from both treatment groups, we used the general linear model to estimate 
activation by palatable stimuli versus purified water. This contrast was tested by permutation at the level of spatially contiguous supra-threshold voxel clusters, controlling the family-wise probability of type 1 error at $P<0.05$ to generate a map of the brain systems activated by food reward. Within this reward system, we separately tested at each voxel the hypotheses that $\Delta$-BOLD $=0$ for GSK1521498 and for NTX, and that $\Delta-$ BOLD $_{\mathrm{GSK} 1521498}=\Delta-\mathrm{BOLD}_{\mathrm{NTX}}$. These exploratory whole-brain analyses are reported at a cluster-wise level of type 1 error $P<0.05$, uncorrected for multiple comparisons.

\section{Results}

\section{Evaluable data and samples}

PET data used in the analysis consisted of 24 baseline (pre-dose) scans (13 for the GSK1521498 group and 11 for the NTX group) and 44 post-dose scans (24 following GSK1521498 and 20 following NTX). Evaluable fMRI data from 17 participants (9 scanned following GSK1521498 and 8 scanned following NTX) were included in the analysis. The treatment groups of participants with evaluable PET or fMRI data were well matched in terms of: age, body mass index, scores on the TFEQ-R18 (ref. 47) and BIS-11 (ref. 48) questionnaires of eating behaviour and impulsivity, the self-rated 'liking' of their preferred palatable drink stimulus at baseline, dose of radioactivity and injected mass of carfentanil during the PET scans (Supplementary Table S1). Neither treatment group demonstrated a significant post-dose reduction in the 'liking' score for the palatable food stimuli (Supplementary Table S1).

\section{Pharmacokinetics}

Plasma exposure parameters are summarised in Table 1 for GSK1521498, NTX and 6- $\beta$-NTX. Representative concentration-time profiles for GSK1521498, NTX and 6- $\beta$-NTX administered in this study are shown in Supplementary Figure S1.

\section{$\left[{ }^{11} C\right]$-carfentanil PET data}

At baseline, $\left[{ }^{11} \mathrm{C}\right]$-carfentanil showed high binding in the striatum, the thalamus and the amygdala (Figure 1). Dose-dependent reductions in regional $\mathrm{BP}_{\mathrm{ND}}$ were observed following the administration of both GSK1521498 and NTX in all ROIs except the occipital cortex, justifying its selection as a reference region (Figure 1).

There were no regional differences in $\mathrm{RO}$ for either drug, so regional occupancies were averaged to estimate global RO. Equation (3) provided a good fit to the data on plasma concentration and RO acquired at all times following a dose of GSK1521498, indicating a direct relationship between exposure and occupancy $^{40,49}$ (Figure 2). We were thus able to estimate the plasma concentration of GSK1521498 associated with $50 \%$ occupancy of the $\mu-\mathrm{OR}$ : $\mathrm{EC}_{50}=7.10 \mathrm{ng} \mathrm{ml}^{-1} \quad(95 \% \quad$ confidence interval $(\mathrm{CI})=5.96-8.25 \mathrm{ng} \mathrm{ml}^{-1}$ ). For NTX and 6- $\beta$-NTX, there was a time-dependent effect (hysteresis) on the exposure-occupancy relationship, that is, a given plasma concentration resulted in greater occupancy at later time points. Hence, the direct model did not provide adequate characterisation of RO based on plasma concentrations of NTX or $6-\beta-N T X$ (Figure 2) and time-independent $\mathrm{EC}_{50}$ estimates could not be determined for either species.

Because of the dependence of the NTX doseoccupancy relationship on the time post dose, the dose-occupancy relationships for NTX and GSK1521498 were estimated using data acquired $<8 \mathrm{~h}$ post dose. The dose required to achieve $50 \%$ RO (effective dose 50) was estimated at $1.50 \mathrm{mg}(95 \%$ $\mathrm{CI}=1.24-1.76 \mathrm{mg}$ ) for GSK1521498. The apparent effective dose 50 for NTX was $5.60 \mathrm{mg}(95 \%$ $\mathrm{CI}=3.65-7.54 \mathrm{mg}$; Figure 1).

\section{fMRI data}

Regional analysis confirmed the main effect of the task with significant BOLD signal increases in regions predicted to be activated by food rewards (Figure 3). Whole-brain mapping demonstrated widespread activation involving these ROIs (as well as some other cortical and sub-cortical regions), thus further justifying their previous selection (Figure 3 and Supplementary Table S4 for anatomical details).

We tested for modulation of food reward-related activation by each drug in each ROI. A significant treatment effect was found only for GSK1521498 and only in the amygdala $(t=-5.9$, d.f. $=8, P=0.0004$; Figure 3 and Supplementary Table S2). GSK1521498 attenuated amygdalar activation in response to palatable stimuli; this effect remained after covarying for between-subject differences in baseline BOLD activation and opioid RO $(t=-3.1$, d.f. $=12, P=0.0085$; Supplementary Table S3). The standardised effect size of GSK1521498 on amygdalar activation (-2.0; $95 \%$ CI -2.7 to -1.2$)$ was greater than the corresponding effect size of NTX $(-0.1 ; 95 \%$ CI -0.9 to 0.7); although the difference in effect sizes (0.6) was not quite significantly different from 0 (95\% CI, -0.04 to $1.3 ; P=0.062$; see Supplementary Tables S2, S3 for details).

Whole-brain mapping demonstrated attenuation of food reward-related activation by GSK1521498 in bilateral amygdala and ventral striatum, as well as thalamus and lateral cortical regions (Figure 4 and Supplementary Table S4 for anatomical details). A similar analysis suggested that different anatomical regions (including the insula and dorsal striatum) were modulated by NTX (Figure 4 and Supplementary Table S4 for anatomical details). A direct comparison between drugs showed that attenuation of food-related activation by GSK1521498 was greater than the effects of NTX in amygdala and ventral striatum bilaterally, whereas effects of NTX were greater than those of GSK1521498 primarily in the insula (Figure 4 and Supplementary Table S4). 


\section{Discussion}

We have combined molecular (PET) and functional (fMRI) neuroimaging techniques in an innovative experimental medicine study comparing the human pharmacology of two OR antagonists, NTX and GSK1521498. We have differentiated the two molecules based on both tissue PK and PD parameters. We
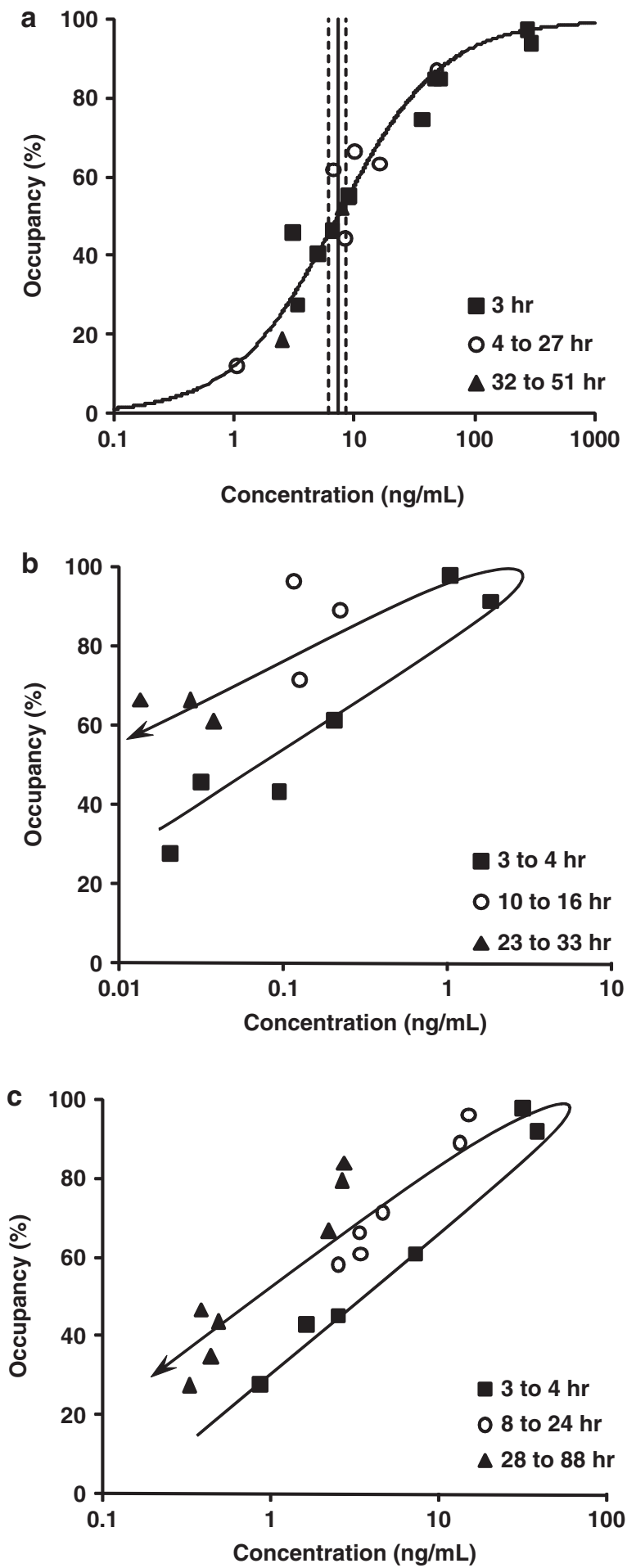

propose that this general approach to integrated neuroimaging may provide a powerful new strategy for early evaluation of the therapeutic potential of new molecules.

\section{Receptor occupancy}

Both drugs dose dependently reduced the specific binding of $\left[{ }^{11} \mathrm{C}\right]$-carfentanil. GSK1521498 demonstrated high affinity for the target, and the relationship between its plasma concentration and $\mu-\mathrm{OR}$ occupancy was time independent. This allowed a simple direct relationship to be defined between plasma concentration and $\mu-\mathrm{OR}$ binding for GSK1521498, distinguishing it from NTX, which demonstrated an indirect relationship between exposure and occupancy. ${ }^{40,49}$ The indirect binding relationship observed for NTX could have several explanations. An oral dose of NTX undergoes rapid and extensive metabolism in humans, producing pharmacologically active metabolites, $6-\beta$-NTX being the predominant one. On the basis of plasma PK and in vitro receptor affinities of 6 - $\beta$-NTX (Table 1, Supplementary Figure S1), it seems likely that a substantial proportion of the target occupancy following an oral dose of NTX represents binding by $6-\beta$ NTX. However, we have modelled the relationship between 6 - $\beta$-NTX plasma concentration and RO over time, and found that this also exhibited an indirect relationship. We cannot exclude the presence of other active metabolites with a different plasma kinetic profile from NTX, which could account for the observed RO time course. Alternatively, NTX or 6- $\beta$ NTX may have long residence times at the $\mu-O R$, or either molecule may not diffuse passively across the blood-brain barrier or may be compartmentalised in the brain parenchyma. Any of these phenomena could result in slow equilibration between the $\mu$-OR and the plasma compartments.

\section{Functional efficacy}

The pharmacological fMRI data indicated that GSK1521498 and NTX modulate different brain regions showing significant fMRI activation responses to palatable food stimuli. We did not find a significant effect of NTX in any of the pre-specified ROIs, whereas GSK1521498 was associated with significant

Figure 2 Relationships between plasma concentration and opioid receptor occupancy for (a) GSK1521498, (b) naltrexone and (c) 6- $\beta$-naltrexol. The timing of individual scans after administration of GSK1521498 or naltrexone is indicated by the shape of the point markers. For GSK1521498, the relationship between plasma exposure and receptor occupancy is independent of time and well fitted by equation (3); the vertical line indicates the effective concentration $50\left(\mathrm{EC}_{50}\right)$ and the dotted lines its $95 \%$ confidence interval. For naltrexone and $6-\beta$-naltrexol, at a given plasma concentration, receptor occupancy is greater for later scans; this hysteresis is highlighted by the arrow which indicates the time ordering of scans. 

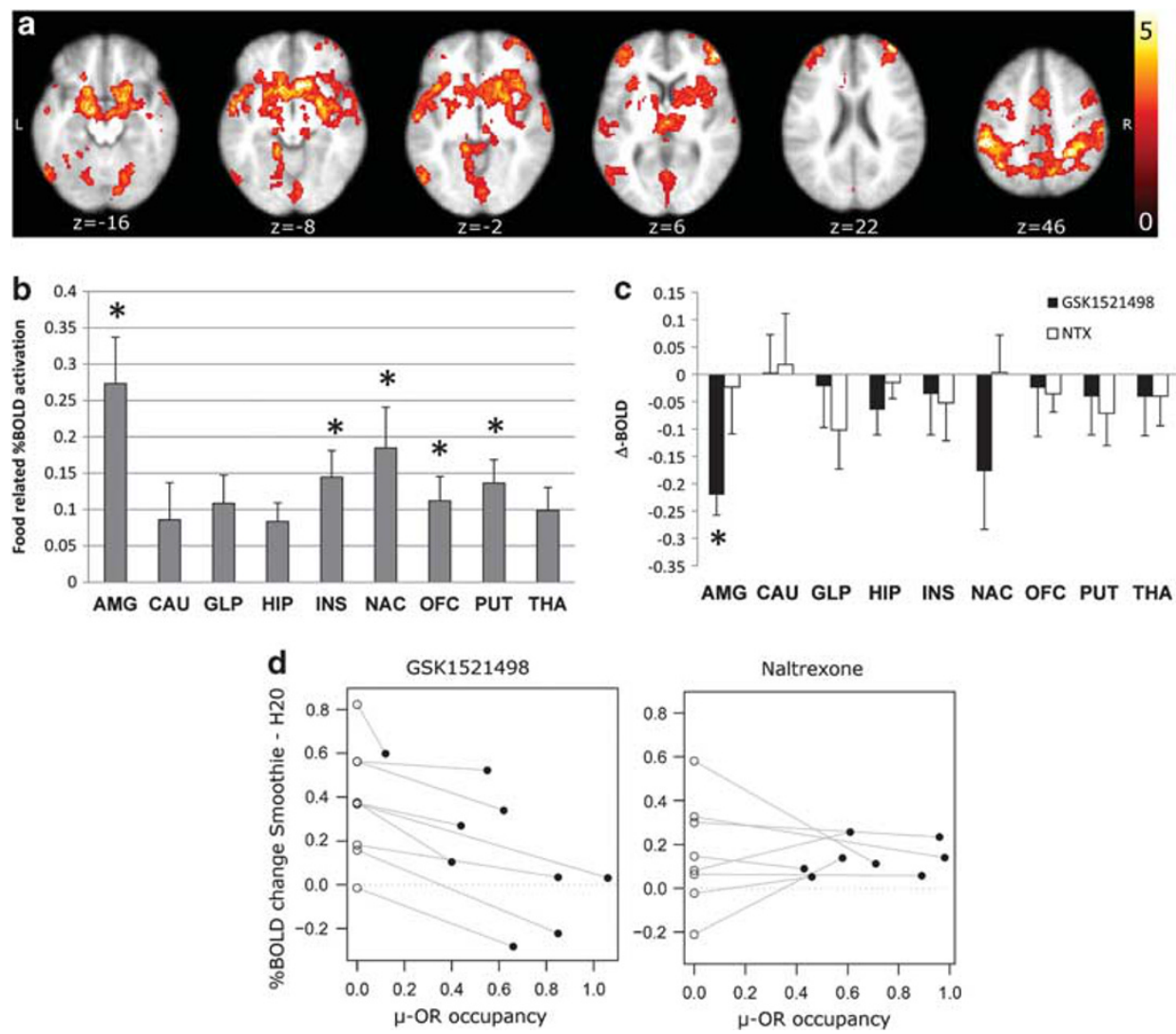

Figure 3 Brain activation by a palatable food stimulus, and the effects of GSK1521498 and naltrexone on food rewardrelated activation in selected regions of interest. (a) Whole-brain map of brain regions activated by experimental contrast between palatable food stimulus and purified water; $z$ indicates distance $(\mathrm{mm})$ superior or inferior to the inter-commissural plane in standard stereotactic space. (b) Bar chart showing magnitude of blood oxygenation level-dependent (BOLD) activation by palatable stimuli versus purified water in nine pre-specified regions of interests: amygdala (AMG), caudate (CAU), globus pallidus (GLP), hippocampus (HIP), insula (INS), nucleus accumbens (NAC), orbitofrontal cortex (OFC), putamen (PUT) and thalamus (THA). (c) Bar chart showing magnitude of change in food-related activation, $\Delta$-BOLD, following treatment with GSK1521498 or naltrexone (NTX) in the same nine regions of interest. (d) Plots of BOLD activation by palatable stimuli versus receptor occupancy from pre- and post-dose scans for each participant in each of the treatment groups. Asterisks $\left({ }^{*}\right)$ denote effects that are significantly different from 0 at $P<0.05$ (Bonferroni corrected). MOR, mu opioid receptor.

attenuation of food-related activation in the amygdala. Whole-brain mapping showed that effects of GSK1521498 were significantly greater on activation in the amygdala and ventral striatum, whereas any effects of NTX were greatest in the insular region. Previous fMRI studies have shown that BOLD activation in the amygdala and ventral striatum can be related to incentive salience and to behaviours reinforced by food or other rewards. ${ }^{39}$ The functional role of the insula is more complex, but it is known to play a role in interoceptive and gustatory processing. ${ }^{50,51}$

Multiple factors could be responsible for pharmacodynamic differences between GSK1521498 and NTX. GSK1521498 has considerably greater selectivity for the $\mu$-OR over the $\kappa-O R$ than either NTX or 6- $\beta$ NTX (Table 1). Pre-clinical studies have indicated that $\kappa-\mathrm{OR}$ and $\mu-\mathrm{OR}$ signalling may have different effects on feeding ${ }^{52,53}$ and reward. Agonism at $\mu$-ORs facilitates dopaminergic neurotransmission in the ventral striatum, which has been specifically implicated in food reward processing. On the other hand, $\kappa-\mathrm{OR}$ agonism tends to reduce dopamine release in the ventral striatum. ${ }^{54,55}$ The reported selectivity of NTX and 6- $\beta$-NTX (Table 1) implies $\kappa$ OR occupancy in the range of $50-80 \%$ at doses which produce $80-90 \%$ occupancy of the $\mu$-OR, making interactions between the two receptor systems relevant for the mode of action of NTX. It may also be relevant that GSK1521498, similar to NTX, can behave as a neutral antagonist or inverse agonist, depending on levels of constitutive activity, whereas 6 - $\beta$-NTX behaves consistently as a neutral antagonist. $^{29,56}$

\section{Methodological issues}

A key strength of the study design is that the PET and fMRI data were acquired from the same participants in the same scanning sessions. This imposed the constraint that the first fMRI scan was always acquired pre-dose and the second scan was acquired post dose, allowing the potential for task repetition 
a

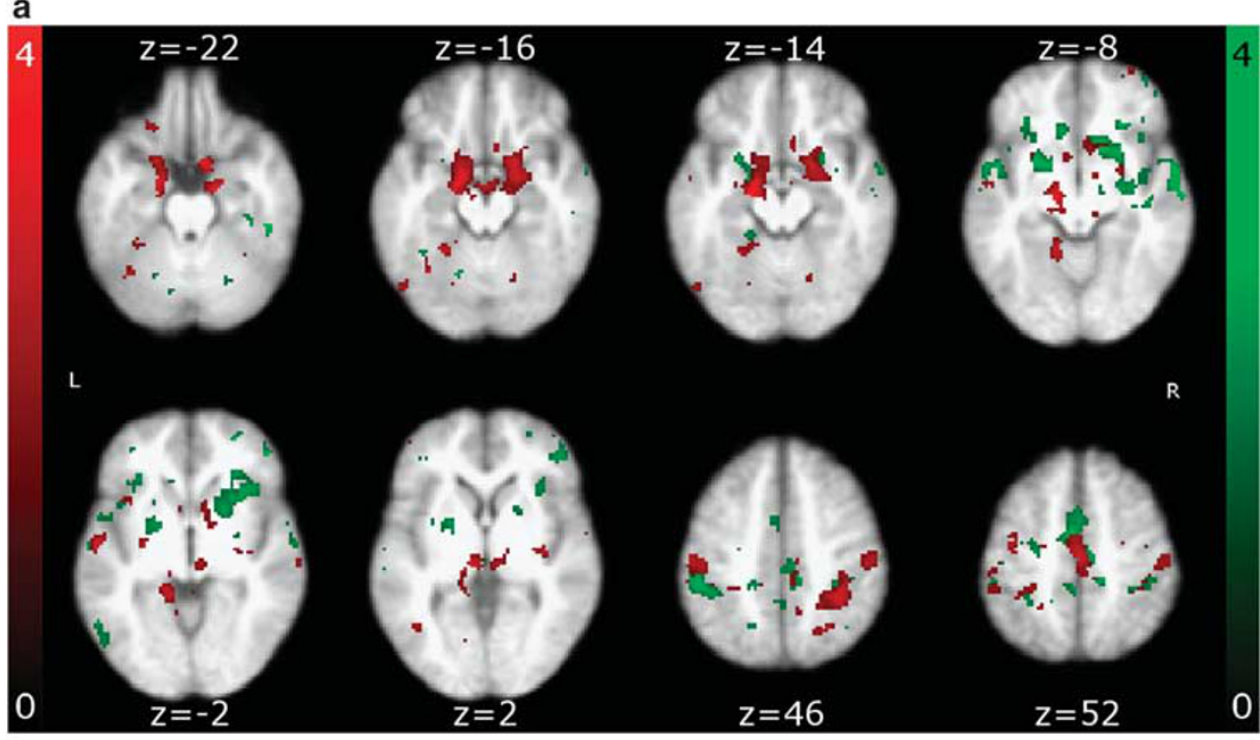

b

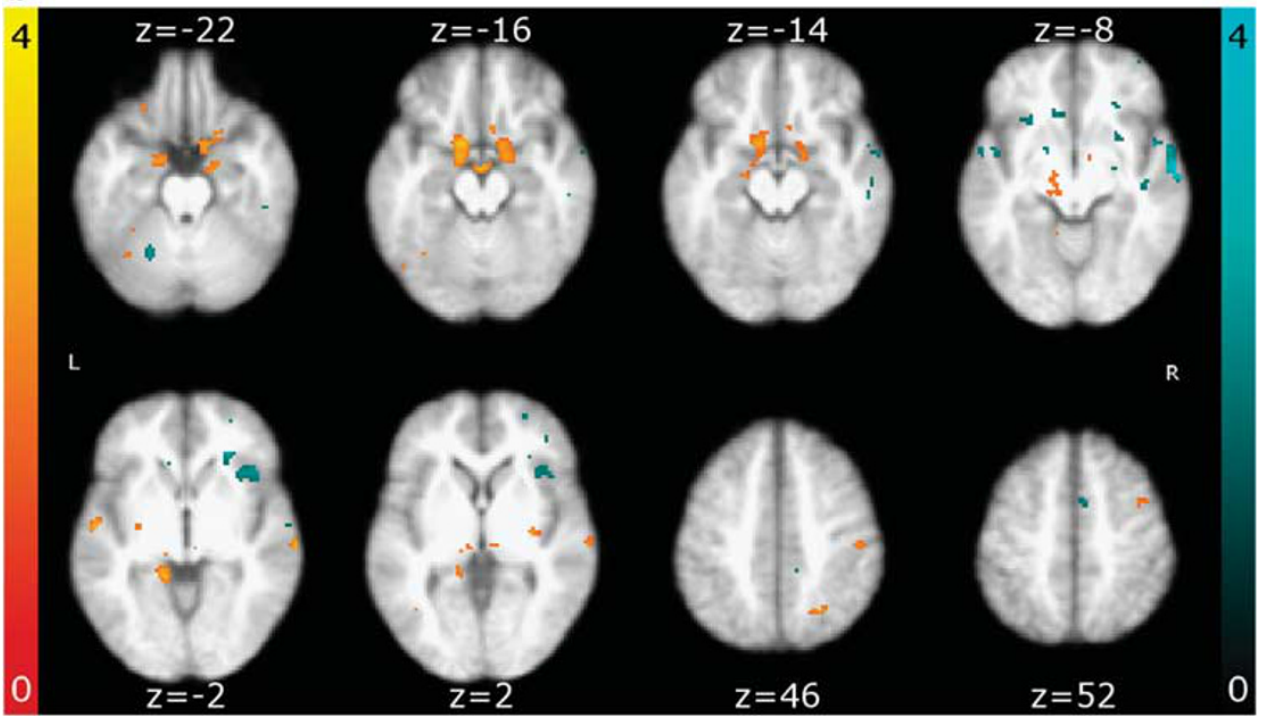

Figure 4 Whole-brain mapping of treatment-related decreases in brain activation by food rewards. (a) Maps of significant change in blood oxygenation level-dependent signal ( $\Delta$-BOLD) in the naltrexone (NTX)-treated group (green voxels) or in the GSK1521498-treated group (red voxels). (b) Map of significant differences in $\Delta$-BOLD between treatment groups: $\Delta$-BOLD $_{\mathrm{GSK} 1521498}>\Delta$-BOLD ${ }_{\mathrm{NTX}}$ (yellow voxels) or $\Delta$-BOLD $\mathrm{NTX}_{\mathrm{NT}}>\Delta-\mathrm{BOLD}_{\mathrm{GSK} 1521498}$ (green voxels). $z$ indicates distance (mm) superior or inferior to the inter-commissural plane in standard stereotactic space.

effects on $\Delta$-BOLD to confound effects of drug treatment. The post hoc analysis of the regional fMRI data demonstrated that greater $\triangle$-BOLD was often associated with greater baseline activation (Supplementary Figure S2). Thus, we cannot entirely exclude the possibility that the treatment difference in fMRI markers may be partly attributable to the interaction between a scanning order effect and sampling variation in baseline activation. However, the effect of GSK1521498 on amygdala activation remains significant after controlling for individual differences in baseline activation.
We chose to activate food reward systems directly by oral administration of a palatable stimulus, rather than by visual presentation of food images, to strengthen the translational link from the experimental data to potentially therapeutic effects of GSK1521498 on food consumption. Because of the radiation risks and costs of PET, the sample size was modest and conferred limited statistical power to detect treatment effects on fMRI measures. Finally, the sample comprised exclusively adult males with normal body weight, body mass index and eating behaviours. OR signalling may differ between sexes, 
and in relation to obesity and eating behaviours, ${ }^{57}$ so our results should be generalised judiciously.

\section{Therapeutic implications}

Drugs that attenuate $\mu$-OR signalling are theoretically likely to be beneficial across a range of disorders of compulsive consumption, marked by habitual or uncontrollable ingestion of food, alcohol, opiates or stimulant drugs. ${ }^{27}$ The novel ligand GSK1521498 has high selectivity for the $\mu$-OR in vitro and high affinity for ORs in vivo. Compared with NTX, we have shown that it has a direct relationship between $\mu$-OR occupancy and plasma concentration, and clearer evidence for functional efficacy in attenuating amygdala activation by food rewards. We hypothesise that these pharmacological differences may be associated with differences in therapeutic efficacy. For example, GSK1521498 may have greater efficacy than NTX in the treatment of maladaptive reward-driven eating behaviours, such as bingeing on energy-dense foods, that are commonly associated with obesity.

\section{Conflict of interest}

All the authors are full-time or part-time employees and share holders of GlaxoSmithKline (GSK). GSK has a commercial interest in GSK1521498 and was the sponsor of this study.

\section{Acknowledgments}

We thank the study participants, the staff of the GSK Clinical Imaging Centre, London, UK and the staff of Hammersmith Medicines Research, for their support in conduct of the study; Wenli Tao and David Collins for statistical advice on study design; and members of the GSK1521498 project team.

\section{Disclosure}

The study was sponsored by GlaxoSmithKline (http:// www.gsk.com) and the protocol was posted on http:// clinicaltrials.gov (NCT00976066) before study initiation. Appropriate ethical committee and regulatory approvals were obtained from the NHS Brent Medical Ethics Committee, Northwick Park Hospital, Middlesex, UK (ref. 09/H0717/30), the Medicines and Healthcare products Regulatory Agency (MHRA Clinical Trial Authorization; EudraCT number 2009010358), and the Administration of Radioactive Substances Advisory Committee, UK Health Protection Agency (ARSAC number RPC 612/3764/24660). All PET and MRI scans were conducted at the GSK Clinical Imaging Centre, Hammersmith Hospital, London, UK. Overnight stays and non-scanning procedures were carried out by Hammersmith Medicines Research, Park Royal, London, UK. Venous plasma samples were analysed at the GlaxoSmithKline Drug Metabolism and Pharmacokinetics laboratories in Ware, UK (for GSK1521498) and in Durham, NC, USA (for naltrexone and 6- $\beta$-naltrexol).

\section{References}

1 Lord JAH, Waterfield AA, Hughes J, Kosterlitz HW. Endogenous opioid peptides: multiple agonists and receptors. Nature 1977; 267: 495-499.

2 Mansour A, Hoversten MT, Taylor LP, Watson SJ, Akil H. The cloned mu, delta and kappa receptors and their endogenous ligands: evidence for two opioid peptide recognition cores. Brain Res 1995; 700: 89-98.

3 Snyder SH, Pasternak GW. Historical review: opioid receptors. Trends Pharmacol Sci 2003; 24: 198-205.

4 Leibowitz SF. Brain neurotransmitters and appetite regulation. Psychopharmacol Bull 1985; 21: 412-418.

5 Morley JE. Neuropeptide regulation of appetite and weight. Endocr Rev 1987; 8: 256-287.

6 Cooper S, Kirkham T. Opioid mechanisms in the control of food consumption and taste preferences. Opioids II. In: Herz A (ed). Handbook of Experimental Pharmacology 1993, Springer-Verlag: Berlin, pp 239-262.

7 Gosnell B, Levine A. Stimulation of ingestive behavior by preferential and selective opioid agonists. Cooper SJ, Clifton PG (eds). In: Drug receptor subtypes and ingestive behavior. 1996, Academic Press: London, UK, pp 147-166.

8 Glass MJ, Billington CJ, Levine AS. Opioids and food intake: distributed functional neural pathways? Neuropeptides 1999; 33: 360-368.

9 Nathan PJ, Bullmore ET. From taste hedonics to motivational drive: central opioid receptors and binge-eating behaviour. Int $J$ Neuropsychopharmacol 2009; 12: 995-1008.

10 Gosnell BA, Levine AS, Morley JE. The stimulation of food intake by selective agonists of mu, kappa and delta opioid receptors. Life Sci 1986; 38: 1081-1088.

11 Bakshi VP, Kelley AE. Feeding induced by opioid stimulation of the ventral striatum: role of opiate receptor subtypes. J Pharmacol Exp Ther 1993; 265: 1253-1260.

12 Shippenberg TS, Elmer GI. The neurobiology of opiate reinforcement. Crit Rev Neurobiol 1998; 12: 267-303.

13 Kelley AE, Bakshi VP, Haber SN, Steininger TL, Will MJ, Zhang M. Opioid modulation of taste hedonics within the ventral striatum. Physiol Behav 2002; 76: 365-377.

14 Figlewicz DP, Sipols AJ. Energy regulatory signals and food reward. Pharmacol Biochem Behav 2010; 97: 15-24.

15 Peciña S, Berridge KC. Hedonic hot spot in nucleus accumbens shell: where do $\mu$-opioids cause increased hedonic impact of sweetness? J Neurosci 2005; 25: 11777-11786.

16 Zhang M, Kelley AE. Opiate agonists microinjected into the nucleus accumbens enhance sucrose drinking in rats. Psychopharmacology 1997; 132: 350-360.

17 Wright CI, Beijer AVJ, Groenewegen HJ. Basal amygdaloid complex afferents to the rat nucleus accumbens are compartmentally organized. J Neurosci 1996; 16: 1877-1893.

18 Azuma S, Yamamoto T, Kawamura Y. Studies on gustatory responses of amygdaloid neurons in rats. Exp Brain Res 1984; 56: $12-22$.

19 Yamamoto T, Shimura T, Sako N, Yasoshima Y, Sakai N. Neural substrates for conditioned taste aversion in the rat. Behav Brain Res 1994; 65: 123-137.

20 Cardinal RN, Parkinson JA, Hall J, Everitt BJ. Emotion and motivation: the role of the amygdala, ventral striatum, and prefrontal cortex. Neurosci Biobehav Rev 2002; 26: 321-352.

21 Kelley AE, Schiltz CA, Landry CF. Neural systems recruited by drug- and food-related cues: studies of gene activation in corticolimbic regions. Physiol Behav 2005; 86: 11-14.

22 Will MJ, Franzblau EB, Kelley AE. The amygdala is critical for opioidmediated binge eating of fat. NeuroReport 2004; 15: 1857-1860.

23 Yeomans MR, Gray RW. Opioid peptides and the control of human ingestive behaviour. Neurosci Biobehav Rev 2002; 26: 713-728.

24 Fantino M, Hosotte J, Apfelbaum M. An opioid antagonist, naltrexone, reduces preference for sucrose in humans. Am J Physiol Regul Integr Comp Physiol 1986; 251(1 Pt 2): R91-R96.

25 Yeomans MR, Wright P. Lower pleasantness of palatable foods in nalmefene-treated human volunteers. Appetite 1991; 16: 249-259.

26 Yeomans MR. Palatability and the micro-structure of feeding in humans: the appetizer effect. Appetite 1996; 27: 119-133. 
27 Drewnowski A, Krahn DD, Demitrack MA, Nairn K, Gosnell BA. Naloxone, an opiate blocker, reduces the consumption of sweet high-fat foods in obese and lean female binge eaters. Am J Clin Nutr 1995; 61: 1206-1212.

28 Drewnowski A, Krahn DD, Demitrack MA, Nairn K, Gosnell BA. Taste responses and preferences for sweet high-fat foods: evidence for opioid involvement. Physiol Behav 1992; 51: 371-379.

29 Wang D, Sun X, Sadee W. Different effects of opioid antagonists on $\mu-, \delta$-, and $\kappa$-opioid receptors with and without agonist pretreatment. J Pharmacol Exp Ther 2007; 321: 544-552.

30 Pelotte AL, Smith RM, Ayestas M, Dersch CM, Bilsky EJ, Rothman $\mathrm{RB}$ et al. Design, synthesis, and characterization of $6 \beta$-naltrexol analogs, and their selectivity for in vitro opioid receptor subtypes. Bioorg Med Chem Lett 2009; 19: 2811-2814.

31 Ko MCH, Divin MF, Lee H, Woods JH, Traynor JR. Differential in vivo potencies of naltrexone and $6 \beta$-naltrexol in the monkey. J Pharmacol Exp Ther 2006; 316: 772-779.

32 Meyer MC, Straughn AB, Lo MW. Bioequivalence, dose-proportionality, and pharmacokinetics of naltrexone after oral administration. I Clin Psychiatry 1984; 45: 15-19.

33 Nathan PJ, O’Neill BV, Bush MA, Koch A, Tao WX, Maltby K et al. Opioid receptor modulation of hedonic taste preference and food intake: a single dose safety, pharmacokinetic and pharmacodynamic investigation with GSK1521498, a novel mu opioid receptor inverse agonist. J Clin Pharmacol 2011 (in press).

34 O’Doherty JP, Deichmann R, Critchley HD, Dolan RJ. Neural responses during anticipation of a primary taste reward. Neuron 2002; 33: 815-826.

35 Filbey FM, Claus E, Audette AR, Niculescu M, Banich MT, Tanabe $\mathrm{J}$ et al. Exposure to the taste of alcohol elicits activation of the mesocorticolimbic neurocircuitry. Neuropsychopharmacology 2008; 33: 1391-1401.

36 Stice E, Spoor S, Bohon C, Veldhuizen MG, Small DM. Relation of reward from food intake and anticipated food intake to obesity: a functional magnetic resonance imaging study. J Abnorm Psychol 2008; 117: 924-935.

37 Petrovic P, Pleger B, Seymour B, Klöppel S, De Martino B, Critchley $\mathrm{H}$ et al. Blocking central opiate function modulates hedonic impact and anterior cingulate response to rewards and losses. J Neurosci 2008; 28: 10509-10516.

38 Myrick H, Anton RF, Li X, Henderson S, Randall PK, Voronin K. Effect of naltrexone and ondansetron on alcohol cue-induced activation of the ventral striatum in alcohol-dependent people. Arch Gen Psychiatry 2008; 65: 466-475.

39 Fletcher PC, Napolitano A, Skeggs A, Miller SR, Delafont B, Cambridge VC et al. Distinct modulatory effects of satiety and sibutramine on brain responses to food images in humans: a double dissociation across hypothalamus, amygdala, and ventral striatum. J Neurosci 2010; 30: 14346-14355.

40 Abanades S, van der Aart J, Barletta JA, Marzano C, Searle GE, Salinas CA et al. Prediction of repeat-dose occupancy from singledose data: characterisation of the relationship between plasma pharmacokinetics and brain target occupancy. J Cereb Blood Flow Metab 2011; 31: 944-952.

41 In British National Formulary, 59, Royal Pharmaceutical Society: London, UK, 2010.

42 Jewett DM. A simple synthesis of $\left[{ }^{11} \mathrm{C}\right]$ carfentanil using an extraction disk instead of HPLC. Nucl Med Biol 2001; 28: 733-734.
43 Tziortzi AC, Searle GE, Tzimopoulou S, Salinas C, Beaver JD, Jenkinson $\mathrm{M}$ et al. Imaging dopamine receptors in humans with [11C]-(+)-PHNO: dissection of D3 signal and anatomy. NeuroImage 2010; 54: 264-277.

44 Innis RB, Cunningham VJ, Delforge J, Fujita M, Gjedde A, Gunn $\mathrm{RN}$ et al. Consensus nomenclature for in vivo imaging of reversibly binding radioligands. J Cereb Blood Flow Metab 2007; 27: 1533-1539.

45 Gunn RN, Lammertsma AA, Hume SP, Cunningham VJ. Parametric imaging of ligand-receptor binding in PET using a simplified reference region model. Neuroimage $1997 ; \mathbf{6}$ : 279-287.

46 Jack Jr CR, Bernstein MA, Fox NC, Thompson P, Alexander G, Harvey $\mathrm{D}$ et al. The Alzheimer's disease neuroimaging initiative (ADNI): MRI methods. J Magn Reson Imaging 2008; 27: 685-691.

47 Karlsson J, Persson LO, Sjöström L, Sullivan M. Psychometric properties and factor structure of the Three-Factor Eating Questionnaire (TFEQ) in obese men and women. Results from the Swedish Obese Subjects (SOS) study. Int I Obes 2000; 24: 1715-1725.

48 Patton JH, Stanford MS, Barratt ES. Factor structure of the Barratt Impulsiveness Scale. J Clin Psychol 1995; 51: 768-774.

49 Derendorf H, Meibohm B. Modeling of pharmacokinetic/pharmacodynamic (PK/PD) relationships: concepts and perspectives. Pharm Res 1999; 16: 176-185.

50 Small DM. Taste representation in the human insula. Brain Structure Func 2010; 214: 551-561.

51 Small DM, Prescott J. Odor/taste integration and the perception of flavor. Exp Brain Res 2005; 166: 345-357.

52 Wolinsky TD, Carr KD, Hiller JM, Simon EJ. Effects of chronic food restriction on mu and kappa opioid binding in rat forebrain: a quantitative autoradiographic study. Brain Res 1994; 656: 274-280.

53 Wolinsky TD, Carr KD, Hiller JM, Simon EJ. Chronic food restriction alters $\mu$ and $\kappa$ opioid receptor binding in the parabrachial nucleus of the rat: a quantitative autoradiographic study. Brain Res 1996; 706: 333-336.

54 Bruijnzeel AW. kappa-opioid receptor signaling and brain reward function. Brain Res Rev 2009; 62: 127-146.

55 Shippenberg TS, Zapata A, Chefer VI. Dynorphin and the pathophysiology of drug addiction. Pharmacol Ther 2007; 116: 306-321.

56 Raehal KM, Lowery JJ, Bhamidipati CM, Paolino RM, Blair JR, Wang $\mathrm{D}$ et al. In vivo characterization of $6 \beta$-naltrexol, an opioid ligand with less inverse agonist activity compared with naltrexone and naloxone in opioid-dependent mice. J Pharmacol Exp Ther 2005; 313: 1150-1162.

57 Bencherif B, Guarda AS, Colantuoni C, Ravert HT, Dannals RF, Frost JJ. Regional $\mu$-opioid receptor binding in insular cortex is decreased in bulimia nervosa and correlates inversely with fasting behavior. J Nucl Med 2005; 46: 1349-1351.

(c) This work is licensed under the Creative Commons Attribution-NonCommercialNo Derivative Works 3.0 Unported License. To view a copy of this license, visit http://creativecommons. org/licenses/by-nc-nd/3.0/

Supplementary Information accompanies the paper on the Molecular Psychiatry website (http://www.nature.com/mp) 